\title{
Method of determining the optimal version of the piezometer of gas distribution networks of medium and high pressure
}

\author{
Viktor Zhila, and Aleksey Klochko* \\ Moscow State University of Civil Engineering, Yaroslavskoe shosse, 26, Moscow, 129337, Russia
}

\begin{abstract}
The article deals with the issues of determining the optimal type of piezometric pressure graph in high-and medium-pressure gas pipelines in the design of gas distribution networks. Along with the most commonly used ways to reduce the cost of building gas distribution networks by finding the optimal configuration of gas pipelines, which give a great economic and environmental effect, it is necessary to rationally use the pressure drop provided to the network. This step in engineering practice allows you to additionally get money savings. The use of various schemes for supplying consumers with gas in cities and villages also has a great economic effect, but modern equipment allows you to make the transition to a single-stage scheme of gas distribution networks for cities. When designing gas distribution networks, the available pressure drop can be used by implementing various technical solutions, but the correct choice of pressures in the nodal points contributes to an economic effect. The designed configuration of the gas distribution network with the adopted type of piezometric graph can ensure reliable operation for a long time. The results obtained are recommended for use in the design of gas distribution networks of cities, other settlements and distribution networks of industrial enterprises
\end{abstract}

\section{Introduction}

Modern gas distribution systems are a complex of structures consisting of low, medium and high pressure networks, gas distribution stations, gas control points and installations. The multi-stage gas distribution system has become widespread. The gas passes sequentially through high-pressure, medium-pressure, and low-pressure gas pipelines [1].

Currently, in European countries and Russia, a single-stage gas distribution system with the installation of combined pressure regulators is increasingly being used. In this case, the combined pressure regulator can be installed in each entrance of a multi-storey building, each house, individual buildings.

\footnotetext{
* Corresponding author: klo4ko_aleksey@mail.ru
} 


\section{Literature review}

The gas distribution scheme becomes flexible: independence of connection of any object is achieved, metal consumption is reduced, investments in construction are reduced by reducing the diameter of gas pipelines. In this case, the distribution gas network is completely designed for medium pressure. When choosing a dead-end gas network of medium pressure, it is necessary to make a technical and economic calculation, in which it is possible to determine the optimal shape of the piezometer, which will contribute to achieving the minimum cost of the gas distribution network.

The choice of the optimal pressure drop for dead-end networks is considered in the works [2, 3], but these recommendations and algorithms affect and are applicable only for low-pressure networks, and there are no recommendations for choosing the optimal form of the piezometric pressure drop graph for high-and medium-pressure networks. In this paper, we will consider this issue. The classical scheme of pressure distribution in a dead-end gas distribution network is shown in Figure 1

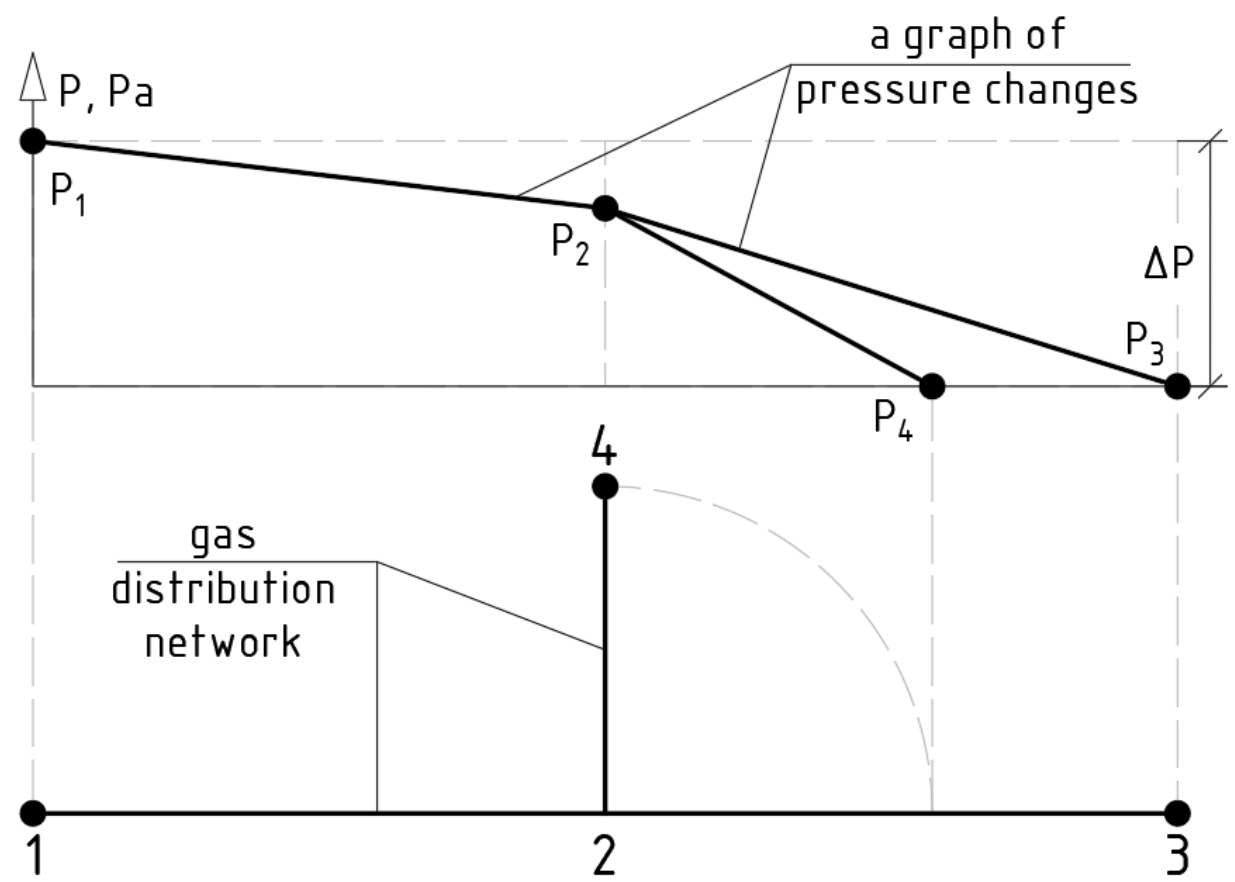

Fig. 1. Pressure change in the gas pipeline

\section{Materials and methods}

When designing gas distribution networks, it is necessary to be guided by both technical parameters: ensuring the required pressure in front of subscribers, sufficient reliability of gas distribution networks and mandatory safety of the subsequent safety of operation of these networks. But it is also very important to use economic parameters in your calculations. An interesting fact is that some technical recommendations can immediately provide economic efficiency. Often, in optimization tasks to improve the efficiency of gas distribu- 
tion systems, they change the connection schemes of subscribers or find the most rational configuration of the gas distribution network [4 - 24]. We will consider the technical and economic calculation of a dead-end gas network of high (medium) pressure and will obtain a mathematical algorithm for determining the optimal shape of the piezometric graph in the design of gas distribution networks. Let's imagine a gas distribution network of a dead-end structure in Figure 2.

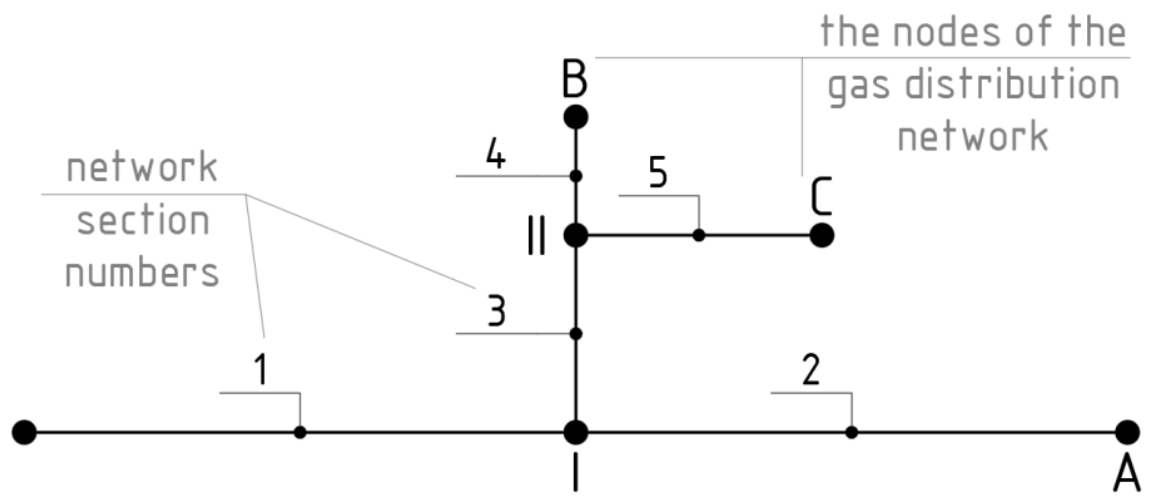

Fig. 2. Diagram of a dead-end gas network of high (medium) pressure

Gas networks are designed for a given pressure drop. The task of technical and economic calculation of dead-end gas networks is reduced to the choice of optimal diameters with a rational distribution of pressure differences between the sequentially connected sections of gas pipelines.

Dead-end gas pipelines are branched networks that feed gas at one point and distribute it to consumers. Any section of an extensive network has a one-way power supply, so the gas consumption for the sections is determined unambiguously. Gas costs for consumers are assumed to be equal $Q_{i}$ and the length of the sections $L_{i}$.

The calculated pressure drop is set $\delta p=p_{b}{ }^{2}-p_{\mathrm{e}}{ }^{2}$. Index " $\mathrm{b}$ "-indicates the initial parameters, and index "e" - the final parameters. Consider the equations that determine the calculation

$$
\begin{array}{r}
\mathrm{K}=b \cdot d l ; \delta p=a \frac{Q^{2}}{d^{5,25}} \cdot l \\
\sum_{1}^{n} k=\sum_{1}^{n} b \cdot a^{0,19} \cdot Q^{0,38} \cdot l^{1,19} \cdot \delta \mathrm{p}^{-0,19} \\
\sum \delta p_{i}-\delta p=0
\end{array}
$$

The number of equations (3) is equal to the number of nodes with specified pressures, that is, the number of end points (points A, B, and C in Fig. 1)

Equations 2 and 3 define the problem, which is reduced to finding the minimum of the function $\sum_{1}^{n} k_{i}$. Let's write a Lagrange function and equate its partial derivatives to zero. We get a system of equations:

$$
\frac{\partial \varphi}{\partial \Delta \mathrm{p}_{\mathrm{i}}}=0
$$

The number of equations is equal to the number of unknowns. We introduce the limiting conditions $4,5,6$.

$$
\begin{aligned}
& \delta \mathrm{p}_{1}+\delta_{2}-\delta \mathrm{p}=0 \\
& \delta \mathrm{p}_{1}+\delta_{3}+\delta_{4}-\delta \mathrm{p}=0 \\
& \delta \mathrm{p}_{1}+\delta_{3}+\delta_{5}-\delta \mathrm{p}=0
\end{aligned}
$$

We will write a Lagrange function: 


$$
\begin{aligned}
& \varphi=\sum_{1}^{5} \mathrm{ba}^{0,19} \cdot \mathrm{Q}^{0,38} \cdot \mathrm{l}^{1,19} \cdot \delta \mathrm{p}^{-0,19}+\lambda_{\mathrm{A}} \cdot\left(\delta \mathrm{p}_{1}+\delta \mathrm{p}_{2}-\delta \mathrm{p}\right)+\lambda_{\mathrm{B}} \cdot\left(\delta \mathrm{p}_{1}+\delta \mathrm{p}_{3}+\right. \\
& \left.\delta \mathrm{p}_{4}-\delta \mathrm{p}\right)+\lambda_{\mathrm{C}} \cdot\left(\delta \mathrm{p}_{1}+\delta \mathrm{p}_{3}+\delta \mathrm{p}_{5}-\delta \mathrm{p}\right)
\end{aligned}
$$

where $\lambda_{\mathrm{A}}, \lambda_{\mathrm{B}}, \lambda_{\mathrm{C}^{-}}$undefined multipliers

Equate the partial derivatives of the function $\varphi$ to zero:

$$
\begin{aligned}
& \frac{\partial \varphi}{\partial \delta \mathrm{p}_{1}}=-\mathrm{ba}^{0,19} \cdot \mathrm{Q}_{1}^{0,38} \cdot \mathrm{l}_{1}^{1,19} \cdot 0,19 \delta \mathrm{p}_{1}{ }^{-0,19}+\lambda_{\mathrm{A}}+\lambda_{\mathrm{B}}+\lambda_{\mathrm{C}}=0 \\
& \frac{\partial \varphi}{\partial \delta \mathrm{p}_{2}}=-\mathrm{ba}^{0,19} \cdot \mathrm{Q}_{2}{ }^{0,38} \cdot \mathrm{l}_{2}^{1,19} \cdot 0,19 \delta \mathrm{p}_{2}{ }^{-0,19}+\lambda_{\mathrm{A}}=0 \\
& \frac{\partial \varphi}{\partial \delta \mathrm{p}_{3}}=-b \mathrm{a}^{0,19} \cdot \mathrm{Q}_{3}{ }^{0,38} \cdot \mathrm{l}_{3}^{1,19} \cdot 0,19 \delta \mathrm{p}_{3}{ }^{-0,19}+\lambda_{\mathrm{B}}+\lambda_{\mathrm{C}}=0 \\
& \frac{\partial \varphi}{\partial \delta \mathrm{p}_{4}}=-b \mathrm{a}^{0,19} \cdot \mathrm{Q}_{4}{ }^{0,38} \cdot \mathrm{l}_{4}^{1,19} \cdot 0,19 \delta \mathrm{p}_{4}{ }^{-0,19}+\lambda_{\mathrm{B}}=0 \\
& \frac{\partial \varphi}{\partial \delta \mathrm{p}_{5}}=-b \mathrm{a}^{0,19} \cdot \mathrm{Q}_{5}{ }^{0,38} \cdot \mathrm{l}_{5}^{1,19} \cdot 0,19 \delta \mathrm{p}_{5}{ }^{-0,19}+\lambda_{\mathrm{C}}=0
\end{aligned}
$$

By subtracting the corresponding equations $8,9,10,11,12$, we exclude undefined factors. From equation 8 , subtract 9,10 , and from equation 10 , subtract equations 11 and 12 .

$$
\begin{aligned}
& Q_{2}{ }^{0,38} 1^{1,19} \delta p_{2}{ }^{-1,19}+Q_{3}{ }^{0,38} l_{3}{ }^{1,19} \cdot \delta p_{3}{ }^{-1,19}-Q_{1}{ }^{0,38} \cdot l_{1}^{1,19} \delta p_{1}{ }^{-1,19}=0 \\
& Q_{4}{ }^{0,38} l^{1,19} \delta p_{4}{ }^{-1,19}+Q_{5}{ }^{0,38} l_{5}{ }^{1,19} \delta p_{5}{ }^{-1,19}-Q_{3}{ }^{0,38} l_{3}{ }^{1,19} \delta p_{3}{ }^{-1,19}=0
\end{aligned}
$$

The number of equations is equal to the number of nodes with undefined pressures. Equation 13 represents the balance of expressions of the type $Q_{i}{ }^{0,38} \cdot l^{1,19} \cdot \delta p^{-1,19}$ for node I, and 14 for node II. If the gas flow approaches the node, then a minus sign is provided before the expression $Q_{i}{ }^{0,38} \cdot l^{1,19} \cdot \delta p^{-1,19}$, if it departs from the node - a plus sign.

\section{Results of research}

These equations allow us to determine the economically optimal pressure differences for each section.

We call it

then for a branched network we have:

$$
A_{i}=Q_{i}^{0,38} \cdot l^{1,19}
$$

$$
\begin{aligned}
& \sum \delta p_{i}=\delta p \\
& \sum A_{i} \delta p_{i}^{-1,19}=0
\end{aligned}
$$

The balances in the nodes are not observed, as shown by equation 18 :

$$
\sum A_{i} \delta p_{i}^{-1,19}=\Delta
$$

where $\Delta$ is the balance discrepancy in the node.

The correction nodal pressures $\Delta \delta p$ are obtained from the equations:

$$
\begin{aligned}
& \mathrm{A}_{2} \delta \mathrm{p}_{2}{ }^{-1,19}+\mathrm{A}_{3} \delta \mathrm{p}_{3}{ }^{-1,19}-\mathrm{A}_{1} \delta \mathrm{p}_{1}{ }^{-1,19}=\Delta_{\mathrm{I}} \\
& \mathrm{A}_{4} \delta \mathrm{p}_{4}{ }^{-1,19}+\mathrm{A}_{5} \delta \mathrm{p}_{5}{ }^{-1,19}-\mathrm{A}_{3} \delta \mathrm{p}_{3}{ }^{-1,19}=\Delta_{\mathrm{II}}
\end{aligned}
$$

By entering the correction nodal pressures $\Delta \mathrm{p}_{\mathrm{I}}$ and $\Delta \mathrm{p}_{\mathrm{II}}$, we obtain:

$$
\begin{aligned}
& A_{2}\left(\delta p_{2}+\Delta p_{\mathrm{I}}\right)^{-1,19}+A_{3}\left(\delta p_{3}+\Delta p_{\mathrm{I}}-\Delta p_{\mathrm{II}}\right)^{-1,19}-A_{1}\left(\delta p_{1}+\Delta p_{\mathrm{I}}\right)^{1,19}=0 \\
& A_{1}\left(\delta p_{4}+\Delta p_{\mathrm{II}}\right)^{-1,19}+A_{5}\left(\delta p_{5}+\Delta p_{\mathrm{II}}\right)^{-1,19}-A_{3}\left(\delta p_{3}-\Delta p_{\mathrm{II}}+\Delta p_{\mathrm{I}}\right)^{-1,19}=0
\end{aligned}
$$

Decomposing the expressions in parentheses in the Maclaurin series we get a system of equations:

$$
\begin{aligned}
& \sum_{\mathrm{I}} A_{i} \delta p_{i}^{-1,19}-1,19 \sum_{\mathrm{I}} \frac{A_{i} \delta p_{i}^{-1,19}}{\delta p_{\mathrm{i}}} \Delta p_{\mathrm{I}}+1,19 \frac{A_{3} \delta p_{3}{ }^{-1,19}}{\delta p_{3}} \Delta p_{\mathrm{II}}=0 \\
& \sum_{\mathrm{II}} A_{i} \delta p_{\mathrm{i}}^{-1,19}-1,19 \sum_{\mathrm{II}} \frac{A_{i} \delta p_{i}^{-1,19}}{\delta p_{i}} \Delta p_{\mathrm{II}}+1,19 \frac{A_{3} \delta p_{3}{ }^{-1,19}}{\delta p_{3}} \Delta p_{\mathrm{I}}=0
\end{aligned}
$$

The values of the correction pressure losses we get the equations: 


$$
\begin{aligned}
& \Delta p^{\prime}=\frac{\sum A_{i} \delta p_{i}{ }^{-1,19}}{1,19 \sum \frac{A_{i} \delta p_{i}^{-1,19}}{\delta p_{i}}} ; \\
& \Delta p^{\prime \prime}=\frac{\sum \Delta P^{\prime}{ }_{\text {cy }}\left(\frac{A \delta p^{-1,19}}{\delta p}\right)_{\text {y.c.y }}}{1,19 \sum \frac{A_{i} \delta p_{i}^{-1,19}}{\delta p_{i}}} ; \\
& \Delta p=\Delta p^{\prime}+\Delta p^{\prime \prime}
\end{aligned}
$$

where $\Delta p^{\prime}$ is the part of the correction without taking into account the influence of neighboring nodes;

$\Delta p^{\prime \prime}$ - part of the correction that takes into account the influence of neighboring nodes;

$\Delta \mathrm{p}_{\text {c.y. }}^{\prime}$ - the first approximation of the correction in the neighboring node.

The value of $\Delta \mathrm{p}_{\text {c.y. }}^{\prime}$ is multiplied by $\left(\frac{\mathrm{A} \delta \mathrm{p}^{-1,19}}{\delta \mathrm{p}}\right)$ of the section that has a neighboring node. The number of terms $\sum \Delta \mathrm{p}_{\text {c.y. }}^{\prime}\left(\frac{\mathrm{A} \delta \mathrm{p}^{-1,19}}{\delta \mathrm{p}}\right)$ is equal to the number of neighboring nodes. When determining the correction, keep in mind that an expression of the type $\frac{A_{i} \delta p_{i}^{-1,19}}{\delta p_{i}}$ is always positive.

\section{Conclusions}

The developed technique of technical and economic calculations of dead-end gas networks of medium and high pressure. This technique allows for the specified pressure differences $P_{b}^{2}-P_{e}^{2}$. to determine the economical diameters with the optimal distribution of pressure differences between successive sections.

As a result of preliminary calculations, the residual in the nodes is obtained. To minimize the discrepancy, we reduce or increase the correction pressures in the nodes to satisfy the economical node equations.

There are many possible piezometers, and the implementation of design solutions for each of them corresponds to a certain cost of a dead-end network of medium and high pressure. This technique of technical and economic calculation of a dead-end gas network of medium (high) pressure makes it possible to find the optimal shape of the piezometer, at which the cost of the network will be minimal.

\section{References}

1. Russia's Energy Strategy for the Period up to 2035. URL: http://www.energystrategy.ru/ (access date: 02.09.2018).

2. A.A. Ionin Gas supply. Moscow (1989)

3. V.A. Zhila Gas supply: textbook for university students in the specialty "Heat and Gas Supply and Ventilation". Moscow (2014)

4. A.V. Laktyun'kin Selection of the degree of ringing of the high-pressure gas distribution network for the medium-sized settlement. (Moscow, 2018)

5. A.K. Klochko Development of the concept of rational design of gas distribution networks by the iterative search method: (Ph.D. thesis in Engineering Science. Moscow, 2012)

6. Gradient methods. URL: https://studfiles.net/preview/960965/(access date: 01.09.2018).

7. V.A. Zhila, A.K. Klochko, E.A. Gusarova Internet Bulletin of Volgograd State University of Architecture and Civil Engineering 3 (23), (2012) 
8. R.G. Arutjunjan Housing construction 11 (2000)

9. K.A. Kuspekov. Omsk scientific bulletin 1 (107), 14-16 (2012)

10. D.A. Ejbozhenko Approximate methods of the Steiner task solution on the oriented columns. (Ph.D. thesis in physical and mathematical sciences St. Petersburg, 2012)

11. M.A. Bagov, V.C. Kudaev International academy of Sciences. 4, 9-14 (2014)

12. V.N. Melkumov, S.V. Chujkin, A.M. Papshickij, K.A. Skljarov Scientific bulletin of the Voronezh state architectural and construction university. Construction and architecture. 2 (38), 41-48 (2015)

13. V.S. Tarasjan, D.O. Ten. Innovative transport 3 (9),29-32 (2013)

14. V.A. Litvinenko, S.A. Hovanskov, V.S. Hovanskova, E.V. Litvinenko. Informatics, computer facilities and engineering education. 4 (28), 9-16 (2016).

15. K.S. Voronin, Je.D. Shabakaeva, D.D. Shabakaev. Problems of functioning of systems of transport materials of the international scientific and practical conference of students, graduate students and young scientists (with the international participation): in 2 volumes. 18-21 (2016)

16. R. G. Arutyunyan Determination of rational strengthening of the reinforced concrete structures, working in the conditions of seismic impacts, by search optimization method. (Ph.D. thesis in Engineering Science. Moscow, 2000)

17. Yu. A. Tabunshchikov, D. V. Koptev, V.A. Zhila, A.K. Klochko, E. B. Soloveva Gas distribution systems efficiency preference / Scientific and technical magazine Vestnik MGSU 8, 222 (2011).

18. K.M. Adeney, M.J. Korenberg. Neural Networks 13/7, 787-799 (2000).

19. C.-Y. Lin, J.-J. Wang.Transactions on Signal Processing. 1, 363-373 (2014).

20. Y. Masatlioglu, D. Nakajima. Theoretical Economics. 3, 701-728 (2013).

21. A. Oddi, A. Cesta, N. Policella, Smith S.F. Journal of Intelligent Manufacturing 1, 1730 (2010).

22. U. Lechowicz, A. Pollak, A. Podgorska, P. Stawinski, M. Oldak, T. Gambin, A. Franke, B.-S. Petersen, M. Firczuk, H. Skarzynski, R. Ploski.Scientific Reports 1, 2543 (2017)

23. R.Zhang, L. Hanzo. IEEE Vehicular Technology Conference Ottawa (2010)

24. T.H. Lin, J.J. Lin, W.J. Peng, J.H. Liu. Computers \& Chemistry. 2, 109-119 (1999) 